\title{
EMBEDDING PROJECTIVE SPACES ${ }^{1}$
}

\author{
BY M. MAHOWALD AND R. JAMES MILGRAM
}

Communicated by Saunders Mac Lane, May 1, 1967

1. Haefliger reduced the question of embedding manifolds in the Euclidian space $R^{m}$ to a homotopy problem in [6]. Since then it has been of some interest to find examples of $n$-manifolds which embed in $R^{2 n-k}$ for a given $k$. In particular great effort has been spent studying embeddings of the various projective spaces. However, the $k$ that were thus obtained were in no cases larger than 5 or 6 (see for example [7], [8], [9]). Our purpose in this note is to indicate the proofs of the theorems that follow.

THEOREM 1. Let $n \equiv 7(8)$; then $\mathrm{RP}^{n}$ (real $n$-dimensional projective space) embeds in $R^{2 n-k}$ where $k \geqq 2\left[\log _{2}(\alpha(n))\right]-1$. (Here $\alpha(n)$ is the number of ones in the dyadic expansion of $n$.)

THEOREM 2. If $n$ is odd and $\alpha(n)$ is greater than $4+2^{i}$, then $\mathrm{CP}^{n}$ (complex projective space) embeds in $R^{4 n-k}$ with $k \geqq 3+i$.

ThEOREM 3. If $\alpha(n) \geqq 11+2^{i}$ then $\mathrm{QP}^{n}$ (quaternionic projective space) embeds in $R^{8 n-k}$ where $k \geqq 5+i$.

The detailed proof of Theorem 1 appears in [5] so in the sequel we will concentrate on giving those modifications which must be made in [5] so as to prove Theorems 2 and 3.

2. A key lemma. Let $M^{n}$ immerse in $R^{2 n-r}$ and set $k(n)=8 s+2^{t}-1$ (where $n+1=\left(2^{4 s+t}\right) c$ with $c$ odd and $0 \leqq t \leqq 3$ ). Then for $n \geqq 3$ we have:

LEMma 2.1. (a) If $n$ is odd there are exactly two isotopy classes of immersions $M^{n} \subseteq R^{2 n}$. One contains an embedding and the other an immersion with a single double point as its only singularity, but both normal bundles have $k$ independent cross-sections where $k=\min (r, k(n))$.

(b) If $n$ is even and $M^{n}$ orientable then there are $Z$ isotopy classes of immersions $M^{n} \subseteq R^{2 n}$ only one of which contains an embedding. The only immersion with a normal field is the embedding, hence the embedding has $r$ normal fields.

REMark. Part $\mathrm{b}$ is false for nonorientable manifolds for all $n$ [4].

Proof. Part a follows from Whitney's well known results [10] on embeddings and immersions in $R^{2 n}$, and a careful study of how one

${ }^{1}$ This research is supported in part by the U. S. Army Research Office (Durham) and the first named author is an Alfred P. Sloan fellow. 
changes the number of double points in an immersion. The details are in [5]. To prove part $b$ note that, according to Hirsch [1], the isotopy classes of immersions are in 1-1 correspondence with the homotopy classes of cross-sections of the stable $(n+1$-dimensional) normal bundle. If $n$ is even the homotopy classes of $n$-plane bundles stably equivalent to the stable normal bundle are classified by $\chi / 2$ (where $\chi$ is the Euler class of the bundle). But in this case $\chi$ is the obstruction to finding a cross-section. Finally, we note that the normal bundle to an embedding $M^{n} \subset R^{2 n}$ must have Euler class equal to zero [3].

For $f: M^{n} \subseteq R^{n+k}$ we denote by $\eta_{f}$ the normal bundle associated to $f$.

CoROLlARy 2.2. If $n$ is even, $M^{n}$ compact and orientable, and if $\nu$ is a subbundle of $\eta_{f}$ for some immersion $f: M^{n} \subseteq R^{2 n-k}$ with $k>0$, then $\eta_{o}$ where $g$ is the embedding $g: M^{n} \subset R^{2 n}$ also contains $\nu$ as a subbundle.

3. Embedding bundles over projective spaces. Using Corollary 2.2 and the immersion results of [2] we can prove:

TheOREM 3.1. (a) If $2 p<\alpha(n)-\alpha(p+1)-3$, then $\eta_{\mathrm{CP}^{q_{C}} \mathrm{CP}^{n}}$ embeds in $R^{4 q}$ where $n=p+q+1$,

(b) If $4 p<\alpha(n)-\alpha(p+1)-10$, then $\eta_{\mathrm{QP}^{q_{c} \mathrm{QP}}}$ embeds in $R^{8 q}$ where $n=p+q+1$.

The proof follows closely the arguments of $\$ 3$ of [5], and in particular the argument following the proof of Lemma 3.2.

THEOREM 3.2. (a) If $n$ is odd then $\mathrm{CP}^{n} \subset R^{4 n}$ with $\alpha(n)$ trivial sections.

(b) If $\alpha(n)>3$, then $\mathrm{QP}^{n} \subset R^{8 n}$ with $\alpha(n)-3$ sections.

This follows directly from the immersion results of [2] together with 2.2 .

4. Double mapping cylinders and the main theorems. Suppose we have spaces $X, Y$, and $Z$ and maps

$$
f: Y \rightarrow Z, \quad g: Y \rightarrow X
$$

then the double mapping cylinder $M(f, g)$ is obtained from the disjoint union $X \cup I \times Y \cup Z$ by identifying a point $(0, y)$ in $I \times Y$ with $f(y)$ in $Z$ and $(1, y)$ with $g(y)$ in $X$. The usual mapping cylinder is obtained by setting $X=Y$ and $g=$ id. We denote it by $M(f)$.

Let $\mathrm{FP}^{n}$ represent either $\mathrm{CP}^{n}$ or $\mathrm{QP}^{n}$. Let $\mathrm{FP}^{q}$ be embedded in $\mathrm{FP}^{n}$ as the set of points whose last $p+1$ homogeneous coordinates are zero (where $n=p+q+1$ ). Embed $\mathrm{FP}^{p}$ in $\mathrm{FP}^{n}$ as the set of points whose first $q+1$ coordinates equal zero. Finally, set $E_{p, q}$ equal to the set of points with (normalized) homogeneous coordinates $\left\langle x_{1}, \cdots\right.$, 
$\left.x_{q+1}, y_{1}, \cdots, y_{p+1}\right\rangle$ where $\sum_{i} x_{i} \bar{x}_{i}=\sum_{j} y_{j} \bar{y}_{j}=1 / 2$. There are evident projections $\pi_{1}: E_{p, q} \rightarrow F P^{p}, \pi_{2}: E_{p, q} \rightarrow F P^{q}$, and we have

LEMMA 4.1. (a) $M\left(\pi_{1}\right)=\eta_{F_{P}^{p} \subset F P^{n}}$,

(b) $N\left(\pi_{2}\right)=\eta_{\mathrm{FP}^{q}} \subset \mathrm{FP}{ }^{n}$,

(c) $M\left(\pi_{1}, \pi_{2}\right)=\mathrm{FP}^{n}$.

Now, when we have spaces given as double mapping cylinders, we can use the following theorem to obtain embeddings.

THEOREM 4.2. Retaining the previous notation let $X$ be a compact, differentiable, $n$-dimensional manifold and assume we have maps $h, T$ so that

(i) $h: X \subseteq R^{l}$ with $\eta_{h}=k \epsilon \oplus \bar{\eta}$ (where $\bar{\eta}$ is some subbundle of $\eta_{h}$ and $\epsilon$ is the trivial line bundle),

(ii) $T: Z \subseteq R^{m}$ is a topological embedding,

(iii) there is a topological embedding $S: M(f) \rightarrow R^{k} \times R^{m}$ so that $S$ restricted to $Z$ is $T$, then there is a topological embedding of $M(f, g)$ in $R^{l+m+1}$.

The proof is contained in [5]; it is similar to the proof of Theorem 1.2 of [9].

REMARK. When $M(f, g)$ is a manifold and we are in the metastable range then Haefliger's theorem [6] shows that we can assume the embedding is differentiable.

Now, using $4.1,3.1$ and 3.2 it is easy to complete the proofs of Theorems 2 and 3 exactly in the manner Theorem 1 is proved in [5].

\section{REFERENCES}

1. M. Hirsch, Immersion of manifolds, Trans. Amer. Math. Soc. 93 (1959), 242276. 482.

2. R. J. Milgram, Immersing projective spaces, Ann. of Math. (2) 85 (1967), 473-

3. J. Milnor, Lectures on characteristic classes, Lecture notes, Princeton Univ., Princeton, N. J., 1951.

4. M. Mahowald, On the normal bundle of a manifold, Pacific J. Math. 14 (1964), 1335-1341.

5. M. Mahowald and R. J. Milgram, Embedding real projective spaces, Ann. of Math. (to appear).

6. A Haefliger, Prolongements de variete dans varietes, Comment. Math. Helv. 36 (1961), 47-82.

7. B. J. Sanderson, Immersions and embeddings of projective spaces, Proc. London Math. Soc. 16 (1964), 135-153.

8. M. Mahowald, On obstruction theory in orientable fiber bundles, Trans. Amer. Math. Soc. 110 (1964), 315-349.

9. - On embedding manifolds which are bundles over spheres, Proc. Amer. Math. Soc. 15 (1964), 579-583.

10. $\mathrm{H}$. Whitney, The self-intersections of a smooth $n$-manifold in $2 n$-space, Ann. of Math. 45 (1944), 220-246. 